\title{
Plant Biotechnology for the Development of Design Starches
}

\author{
María Victoria Busi, Mariana Martín and Diego F. Gomez-Casati
}

Additional information is available at the end of the chapter

http://dx.doi.org/10.5772/50195

\section{Introduction}

Obesity is a major public health problem due to its pronounced increase and prevalence worldwide. The World Health Organization indicated that in 2005 at least 1.6 billion of adult people were overweight and about 400 million of adults were obese. Predictions for 2015 are even more alarming because indications are that more than 700 million of people will be obese. One of the most common problems associated with obesity is the current lifestyle. Overweight is one of the main risk factors in the development of many chronic diseases, such as respiratory and heart diseases, type 2 diabetes, hypertension and some types of cancer. The increased risk of acquiring some of these diseases is associated with small changes in weight but it can be prevented if appropriate changes in lifestyle are introduced [5].

Furthermore, gastrointestinal infections remain a major health problem despite new advances in medicine. The global incidence of deaths caused by this type of disease is about of 3 million deaths per year. Although this problem is more severe in developing countries, it also occurs in industrialized countries where the incidence of intestinal infection affects about $10 \%$ of the population. In most people, the enteropathogenic bacteria cause gastroenteritis that can be treated with drugs and an adequate rehydration. However, in populations such as old people, children, people with chronic intestinal inflammation and immunodeficiencies, it could be a serious problem, leading to the production of septicemia and death. The control of intestinal infections with antibiotics has been one of the medical breakthroughs of the twentieth century. However, the misuse and abuse of these compounds, has led to increased bacterial resistance. Thus, it becomes extremely important to look for new strategies to prevent and/or treat infections. One promising approach is based on the modulation and control of the intestinal microflora through the diet [6]. 
Starch is a substantial component of the human diet, mainly in populations that are fed on agricultural crops, providing about $50 \%$ of daily energy uptake, mostly through unrefined cereals. In contrast, in westernized societies, the average consumption of grains is much lower, reaching only $25 \%$. Polysaccharides such as glycogen or starch are some of the polymers which can be digested by the enzymes of the human gut. This digestion occurs in the small intestine, except a portion named resistant starch (RS) which is degraded in the large intestine. RS is defined as the set of starch and products of starch degradation (oligosaccharides and others) that are not absorbed in the small intestine but are fermented in the colon producing short-chain fatty acids (such as butyrate), and promotes the normal function of the colonocytes. Because the good function of the human gut is given by the consumption of foods rich in starch, and the change of dietary habits towards healthier eating is not a simple job, the enrichment of some foods with RS becomes the most promising option for a healthy diet $[3,7,8]$.

RS function as dietary fibers, including pre-biotic effect on colon microflora, altering lipid metabolism, improving cholesterol metabolism, and reducing the risk of ulcerative colitis and colon cancer. Since RS is not digested in the small intestine it also reduces the glycemic index of the food [9] (Table 1).

\begin{tabular}{|l|l|}
\hline POTENTIAL PHYSIOLOGICAL EFFECTS & \multicolumn{1}{|c|}{ POSSIBLE PROTECTIVE EFFECTS } \\
\hline Prebiotic and improved bowel health & $\begin{array}{l}\text { Colonic health; colorectal cancer, } \\
\text { inflammatory bowel disease, } \\
\text { constipation, ulcerative colitis }\end{array}$ \\
\hline $\begin{array}{l}\text { Improve insulinaemic and glycaemic } \\
\text { responses }\end{array}$ & $\begin{array}{l}\text { Diabetes, the metabolic syndrome, } \\
\text { impaired insulin and glucose responses }\end{array}$ \\
\hline Improvement of blood lipid profile & $\begin{array}{l}\text { Lipid metabolism, cardiovascular } \\
\text { disease, the metabolic syndrome }\end{array}$ \\
\hline $\begin{array}{l}\text { Increased satiety and synergistic interactions } \\
\text { with other dietary components }\end{array}$ & $\begin{array}{l}\text { Obesity, improved metabolic control and } \\
\text { enhanced bowel health }\end{array}$ \\
\hline $\begin{array}{l}\text { Adjunct to oral rehydration therapies and } \\
\text { increased micronutrient absorption }\end{array}$ & $\begin{array}{l}\text { Treatment of chronic diarrhea and } \\
\text { cholera; osteoporosis }\end{array}$ \\
\hline Thermogenesis & Diabetes and obesity \\
\hline
\end{tabular}

Table 1. Physiological effects of resistant starch (adapted from [3])

There are at least four mechanisms by which resistant starches are obtained [7,10]: RS1: physically inaccessible starch, usually encapsulated in indigestible tissues (encapsulated or embedded within a matrix of lipid and/or protein) ; RS2: starch granules resistant to degradation, with two subtypes, RS2a with low amylose $(0-30 \%)$, which generally loses its strength when cooked, and RS2b, starches with high amylose content which retains its granular structure during processing, RS3: starch retrograde which requires cooking to be released from the granules, and the starch retrograde capacity is affected by the intrinsic biosynthetic process; finally, RS4: chemically modified starches; although this mechanism is the most used to produce resistant starch, there are no reports of changes in plant that can 
mimic those obtained by chemical methods. Because each of these processes is independent, it is possible that in some foods resistant starches are derived from more than one mechanism. In these classes, RS1, RS2 and RS3 can be influenced by genetic manipulation of plants. The high amylose starches have the greatest potential to generate resistant starch through two mechanisms, RS2b and RS3 [11]. To achieve this, three strategies have been proposed: reduction of branching enzyme activity; reduction of the amylopectin synthesis rate without altering the synthesis of amylose or/and the increment of the amylose synthesis without altering the synthesis of amylopectin (Table 2).

\begin{tabular}{|l|l|l|}
\hline $\begin{array}{l}\text { TYPE OF RESISTANT } \\
\text { STARCH }\end{array}$ & $\begin{array}{l}\text { EXAMPLES OF } \\
\text { OCCURRENCE }\end{array}$ & $\begin{array}{l}\text { RESISTANCE REDUCED } \\
\text { BY }\end{array}$ \\
\hline ins1: Physycally & $\begin{array}{l}\text { Whole or partly milled } \\
\text { grains and seed, legumes, } \\
\text { pasta }\end{array}$ & Chewing, milling \\
\hline RS2: Resistant granules & $\begin{array}{l}\text { Raw potatoes, green } \\
\text { bananas, high-amylose } \\
\text { starches, some legumes. } \\
\text { Ungelatinised resistant } \\
\text { granules, hydrolysed slowly } \\
\text { by } \alpha \text {-amylases }\end{array}$ & $\begin{array}{l}\text { Cooking and food } \\
\text { processing }\end{array}$ \\
\hline RS3: Retrograded & $\begin{array}{l}\text { Cooked and cooled potato, } \\
\text { food products with repeated } \\
\text { and/or prolonged heat } \\
\text { treatment, bread, cornflakes }\end{array}$ & Processing conditions \\
\hline RS4: Chemically modified & $\begin{array}{l}\text { Modified starches due to } \\
\text { cross-bonding with esters, } \\
\text { ethers, etc. Some cakes, } \\
\text { breads and fibre-drinks that } \\
\text { were made with modified } \\
\text { starches. }\end{array}$ & $\begin{array}{l}\text { Less susceptible to } \\
\text { digestibility in vitro }\end{array}$ \\
\hline
\end{tabular}

Table 2. Nutritional classification of resistant starches (adapted from [3] and [7]).

Given that each of these mechanisms is independent, it is possible that any food could contain RS derived from more than one mechanism. Moreover, RS, RS1, RS2, and RS3 content in foods can be modified by crop genetics [10]. Examples of major components of dietary RS are retrograded amylose (RS1), such as cooked and cooled starchy foods like pasta salad, and native starch granules (RS2), such as those found in high amylose maize starch and bananas [12]. On the other hand, RS3 preserves its nutritional functionality during the cooking process. Thus, it may be used as a food ingredient. RS3 is produced in two steps: gelatinization, which is a disruption of the granular structure by heating with excess of water [13] and retrogradation, a slow recrystallization of the starch molecules upon cooling or dehydration [14]. The resistant fraction may be then isolated using amylolytic enzymes such as pancreatic amylase [15], or Termamyl-heat stable $\alpha$-amylase [16]. It has 
been shown that the later approach leads to formation of very thermally stable RS3, and to yields up to $40 \%[9,14]$.

Finally, but not least, is the role of investigation and development conducted by researchers from universities and industry. The incorporation of progress in science and the use of currently existing technology contributes to the production of healthy foods, and in this context, designing plants with biology tools to improve their current molecular nutritional qualities is a challenge [5].

The first use of transgenesis in plants in the 1980s brought the arrival of a powerful tool for the study of metabolic regulation and crop improvement. Of particular interest from a health and commercial viewpoint was the potential for increasing yield making alteration of carbon partitioning between sucrose, starch and amino acids [17]. Since that time, plant biotechnology and its commercialization are in exponential phase. Already In 1998, more than 28 million hectares of transgenic crop plants were grown worldwide. Of these 28 million hectares, the largest area was in the USA (22 million hectares) followed by Canada (1.8 million hectares), Argentina (1.8 million hectares) and China (estimated at 1.1 million hectares). It was also estimated that in the US $40 \%$ of the cotton, $24 \%$ of corn and $40 \%$ of soybean planted was transgenic [18].

Given the large amount of information available from molecular biology studies and from genomic programs about the starch biosynthetic genes from crop plants, it is now relatively simple to identify the changes at the DNA level to generate desired starch phenotypes [1921]. Transgenic approaches to altering the composition of crop plants involve two general approaches: overexpression of an endogenous or foreign gene in the target tissue, and use of RNAi technology to specifically suppress the activity of a specific plant gene [10]. We propose in this chapter to give an overview of starch synthesis to review the potential target technologies and to summerize the successful work done by numerous research groups in different plant species using different strategies.

\section{Overview of the starch biosynthesis and degradation in plants}

Polyglucans are the most important and widespread carbohydrate storage compounds found in nature, with glycogen and starch being the most abundant forms. Both polysaccharides are comprised of glucose chains linked by an $\alpha-(1,4)$ bond, and branched at $\alpha-(1,6)$. Glycogen is a homogeneous water-soluble polymer with relatively uniformly distributed branches [22] and is found in organisms such as archaea, bacteria and certain eukaryotes. Starch is made up of amylose (a largely unbranched, minor component) and amylopectin (an asymmetrically branched major component) and is present in the cytoplasm of Rhodophyceae (red algae) and Glaucophyta [23], but is confined to the plastid stroma (chloroplasts in green tissues and amyloplasts in reserve organs) in green algae and higher plants. In fact, starch synthesis is restricted to the Archaeplastida, whose origins are thought to be via a single endosymbiotic event involving ancestors of cyanobacteria and a heterotrophic host [24], rendering the organelle known as the plastid, which is capable of oxygenic photosynthesis. Recent phylogenetic studies indicate that the plastidial starch 
pathway is complex, and made up of genes with both cyanobacterial and eukaryotic origins $[25,26]$, and is in sharp contrast to the lower-complexity pathway of cytosolic starch synthesis found in the Rhodophyceae and Glaucophyta [27]. Phylogenetic analysis of the enzymes of the starch biosynthetic pathway strongly suggests that the pathway was originally cytosolic (in the common ancestor of the Archaeplastida), and then re-directed to plastids via three discrete steps, leaving some enzymes involved in the metabolism of maltooligosaccharides (MOS) and amylopectin degradation in the cytoplasm. The three evolutionary steps involved are: (1) plastidial synthesis of unbranched MOS; (2) glycogen synthesis (including priming steps and branching activities); and (3) plastidial starch synthesis, resulting in the eventual loss of cytosolic starch synthesis. Interestingly, the relocation of the starch synthesis pathway to plastids coincides with the evolution of lightharvesting complexes [26,28].

There are four biochemical steps in each tissue that are required for the synthesis of starch, substrate activation, chain elongation, chain branching, and chain debranching [10] and it involves at least three enzymes such as ADP-glucose pyrophosphorylase (ADPGlc PPase, EC 2.7.7.27), starch synthase (SS, EC 2.4.1.21), and branching enzyme (BE, EC 2.4.1.18) [29,30] (Figure 1).

The first step of the starch biosynthesic pathway is the synthesis of the activated monomer ADPglucose (ADPGlc) from glucose-1-phosphate and ATP, synthesized by ADPGlc PPase. This reaction is the key step for the control of carbon flux through the starch biosynthetic pathway $[29,30]$.

The second step of the starch biosynthesis pathway is the reaction catalyzed by starch synthase, in which the glucosyl moiety of ADPGlc is transferred to the non-reducing end of a pre-existing $\alpha-1,4$ glucan polymer [10]. To date, five SS isoforms have been described based on sequence similarities: granule-bound SS (GBSS), involved mainly in amylose synthesis and the soluble isoforms: SSI (involved in the synthesis of small chains of amylopectin), SSII and SSIII (with a major role in amylopectin synthesis) and SSIV (recently found to be involved in the control of starch granule number and starch granule initiation) [31-34].

To produce an efficient clustering of the branch points and the formation of crystalline lamella, several debranching enzymes (DBE) are required [35,36]. In addition, the degradation of the crystalline granules depends on a recently discovered group of enzymes - the glucan, water dikinases (GWDs) - which phosphorylate crystalline sections of the granules. Such phosphorylation is catalyzed by two GWD types: the GWD1, involved in the tagging of the glucan chains by C-6 phosphorylation, which is a prerequisite for subsequent C-3 phosphorylation by the second isoform, the GWD3/PWD (glucan, water dikinase 3/phosphoglucan, water dikinase) [37-39]. These enzymes seem to have evolved concomitantly with the appearance of starch deposition $[36,40]$.

A fourth obligatory step in starch biosynthesis has been identified through genetic studies but is poorly understood in terms of the biochemical mechanism that mediates the effect. This step is the cleavage of $\alpha-1,6$ linkages by isoamylase-type DBE [10,41]. The DBE are 
crucial for the generation of longer, clustered linear segments in the amylopectin molecule that can crystallize and increase the density of the polysaccharide [42]. Plants contain four DBE genes, three of which are classified as isoamylases on the basis of their sequence homologies and substrate specificities, and one pullulanase-type debranching enzyme $[10,43]$.

While the steps leading to the synthesis of starch are common in most cereals, there are differences in the location and engagement of enzymes, depending on whether the synthesis is in leaf or endosperm (Figure 1).

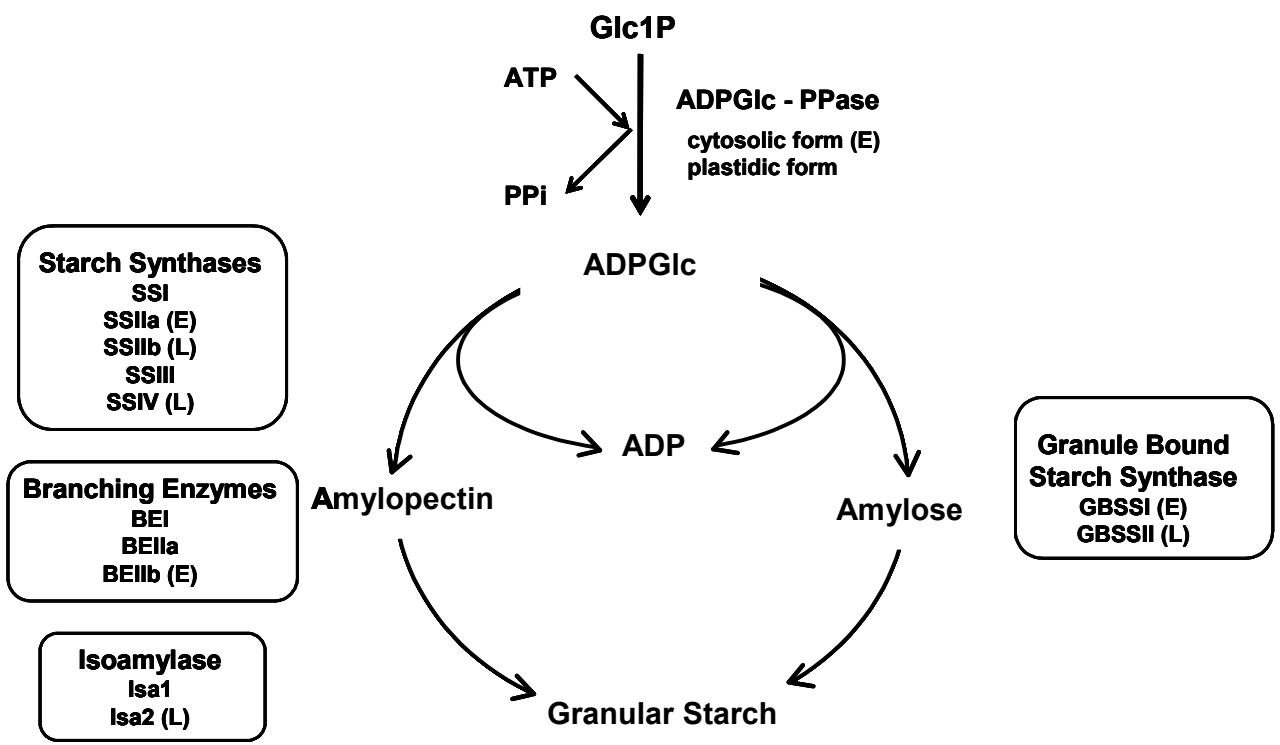

Figure 1. Starch biosynthesis pathway in plants from Glc1P. The scheme indicates the involvement of different isoforms in cereal leaf $(\mathrm{L})$ or endosperm (E). When not specified, the enzymes are dual localized in both compartments (adapted from [10] and [44]).

\section{Carbohydrate binding modules}

Many of the enzymes involved in the pathway of polysaccharide biosynthesis present a carbohydrate binding domain in its structure. The first carbohydrate binding domain described was a cellulose-binding domain [45-47]; but later it has been found other modules in related enzymes that bind polysaccharides other than cellulose. These findings compelled to redefine the nomenclature of these domains, and now are called CBM (carbohydrate binding module). To date over 300 putative sequences in more than 50 different species have been identified, and binding domains have been classified into 64 families based on amino acid sequence, the substrate binding specificity and structure [48] (see Carbohydrate-Binding Module Family Server, http://afmb.cnrsmrs. $\mathrm{fr} / \sim$ pedro/CAZY/cbm.html). 
CBMs have been found in several non-hydrolytic and hydrolytic proteins. Those with hydrolytic activity, such as cellulases, have a complex molecular structure comprised of discrete modules (one catalytic domain and one or more CBMs) that are normally linked by unstructured sequences. The CBMs increase the speed of enzymatic reactions by conducting the catalysis in a close and prolonged physical association with substrates [48] The CBMs present in non-hydrolytic proteins constitute a subunit of the catalytic domain hosts generating cohesive multienzyme complex, which lose enzymatic activity when the CBMs are removed from the structure [48]. Therefore, the CBMs have three general roles with respect to the function of their cognate catalytic modules: (i) a proximity effect, (ii) a targeting function and (iii) a disruptive function [49].

The SBD (starch binding domain) is usually a distinct sequence-structural module that improves the efficiency of an amylolytic enzyme, improving the binding to starch and its hydrolysis. Because this module was first recognised in amylases and thus revealed to cope with raw starch, it was named the raw (granular) starch binding site [50]. At the present, due to the occurrence of SBDs in a wide spectrum of non-amylolytic enzymes, it has become logical to expect a more variable function of these modules. However, there is little evidence that SBD could bind polysaccharides different to starch, although the ability of pure starch binding and degrading seems to be reserved for microorganisms [50,51].

The CBMs have been clasified in ten families based on sequence comparison: (i) CBM20, such as the C-terminal SBD from Aspergillus niger glucoamylase; (ii) CBM21, located at the $\mathrm{N}$-terminal domain in amylase proteins; (iii) CBM25, containing one (i.e. $\beta$-amylase from Bacillus circulans) or two (i.e. Bacillus sp. $\alpha$-amylase) domains; (iv) CBM26, mainly organized in tandem repeats (i.e. C-terminal domains from Lactobacillus manihotivorans $\alpha$-amylase); (v) CBM34, present in the N-terminal domains of neopullulanase, maltogenic amylase and cyclomaltodextrinase; (vi) CBM41, N-terminal SBD, present mostly in bacterial pullulanases; (vii) CBM45, originating from eukaryotic proteins from the plant kingdom (i.e. N-terminal modules of $\alpha$-glucan water dikinases and $\alpha$-amylases); (viii) CBM48, which display glycogen-binding properties (including SBD from the GH13 pullulanase and regulatory modules of mammalian AMP-activated protein kinase); (ix) CBM53, SBD modules from SSIII and (x) CBM58, find in $\alpha$-amylase/neopullulanase of Bacteroides thetaiotaomicron showing maltoheptaose binding [52-54] (http://www.cazy.org). This modules becomes important in breaking down the structure of the substrate due to the presence of two polysaccharide-binding sites [55].

Using bioinformatics techniques several SBDs and several sequences exhibiting similarities to SBDs have also been recognised in enzymes and proteins that are not necessarily amylases such as dual-specific phosphatases. These enzymes should deserve special attention because of their participation in various important physiological processes in plants and mammals. It is worth mentioning that in plants these processes concern starch metabolism, whereas in mammals they participate in the metabolism of glycogen $[56,57]$. The presence of an SBD motif in protein phosphatases reflects their regulatory function since they are involved in polysaccharide metabolism indirectly via modulation of activity of degradative enzymes (i.e. also amylases), such as isoamylase, $\beta$-amylase and 
disproportionating enzyme [85]. In particular, the initial steps of starch degradation at the granule surface are regulated mainly by phosphorylation [50,51]. Furthermore, a starch biosynthetic enzyme, the starch synthase III (SSIII) from Arabidopsis thaliana (AtSSIII) has been reported by our group to have a regulatory role in the synthesis of transient starch [33]. This enzyme contains 1025 amino acid residues and has an N-terminal transit peptide for chloroplast localization followed by three in tandem starch-binding domains (SBD D1, D2 and D3, residues 22-591), which bind to raw starch and its individual components, amylose or amylopectin [53,54,58,59]. The adsorption experiments show that the SBD123 region binds preferentially to amylose, and that the D1 domain is mainly responsible for this selective binding. The D2 domain contains two binding sites including amino acid residues Y394 (binding site 1) and W366 (binding site 2) which act in cooperation with the D1 domain in the binding activity while G335 and W340 have a minor role [54]. It is worth mentioning that our work was the first report on the existence of an SBD in a synthesizing enzyme (AtSSIII) and the first experimental evidence of its starch binding capacity.

\section{Altering the composition and amount of starch by biotechnological manipulation of enzymes}

The alteration of starch quantity and quality can be achieved through the overexpression of some enzymes involved in starch synthesis [60], by mutations or RNAi technology, such as the inhibition of potato SSII, SSIII and GBSS [61], or the decrease in the expression of wheat BEIIa and BEIIb [62,63]. In this way, by affecting the catalytic activity of enzymes involved in the synthesis of amylose or amylopectin, it could be possible to obtain starches for different purposes. Table 3 presents a summary of some of the varieties of plants (transgenic, mutant or silenced by RNAi) that exhibit altered levels of amylose.

The production of high amylose starch is of particular interest because its amount is correlated with the amount of RS in food. Foods with higher content of RS have the potential to improve human health and lower the risk of serious noninfectious diseases. As described above, the amylose content can be increased by the inactivation of the enzymes involved in amylopectin synthesis. In this way, RNAi was used to down-regulate the two different isoforms of starch-branching enzyme (BE) II (BEIIa and BEIIb) in wheat endosperm. Whereas the inhibition of BEIlb expression alone had no effect on amylose content; the decrease of both, BEIIa and BEIlb expression, resulted in the accumulation of starch containing more than $70 \%$ of amylose. When this high amylose starch was used to feed rats as a whole meal, it was observed that short-chain fatty acids such as butyrate, propionate and acetate increased with respect to controls. Short chain fatty acids are derived from the anaerobic fermentation of polysaccharides in the large intestine and are important in improving colonic health. These results indicate that this high-amylose wheat has a significant potential to improve human health through its RS content [62].

The decrease of BEIIb enzyme activity in rice is also traditionally associated with elevated amylose content, increased gelatinization temperature, and a decreased proportion of short amylopectin branches. To further elucidate the structural and functional role of this enzyme, 
the phenotypic effects of down-regulating BEIIb expression in rice endosperm were characterized by Buttardo and coworkers [64] by artificial microRNA (amiRNA) and hairpin RNA (hp-RNA) gene silencing. The results showed that RNA silencing of BEIIb expression in rice grains did not affect the expression of the other major isoforms of BE or SS proteins. The increase in about 2-fold of amylose content was not due to an increase in the relative proportion of amylose chains but instead was due to significantly elevated levels of long and intermediate chains of amylopectin. Rice altered by the amiRNA technique produced a more extreme starch phenotype than those modified using the hp-RNA technique, with a greater increase in the proportion of long and intermediate chains of amylopectin. The major structural modifications of starch produced in the amiRNA lines led to more severe alterations in starch granule morphology and crystallinity as well as digestibility of freshly cooked grains [64].

\begin{tabular}{|l|l|l|l|}
\hline LINES & AMYLOSE (\%) & EVENT & $\begin{array}{l}\text { ENZYME } \\
\text { INVOLVED }\end{array}$ \\
\hline Standard maize & $20-30$ & reference & NA \\
\hline Sugary-1 & 37 & mutation & Isoamylase \\
\hline Dull & 31 & mutation & SSIII \\
\hline Amylose extender & 56 & mutation & BEIIb \\
\hline Indica rice & 27 & reference & NA \\
\hline Japonica rice & 16 & mutation & SSIIa \\
\hline OsSSIIIa & 8 & $\begin{array}{l}\text { retrotransposon } \text { or } \\
\text { mutagenesis }\end{array}$ & SSIIIa \\
\hline OsSSI & 60 & mutation & SSI \\
\hline Standard barley & $20-30$ & reference & NA \\
\hline Waxy & 9 & mutation & GBSS \\
\hline Himalaya 292 & 71 & mutation & SSIIa \\
\hline BEIIa + BEIIb & 75 & RNA-silencing & BEIIa/BEIIb \\
\hline Wheat & $18-36$ & reference & NA \\
\hline Sgp-1 triple null & $31-38$ & mutation & SSIIa \\
\hline BEIIa + BEIIb & 70 & RNAi & BEIIa/BEIIb \\
\hline Potato & 29 & reference & NA \\
\hline BE-II & 38 & antisense & BEII \\
\hline BEI + BEII & $77-87$ & antisense & BEI/BEII \\
\hline
\end{tabular}

Ref: NA, not applicable

Table 3. Amylose content of different lines (wt, mutant and/or transgenic). Adapted from [10].

The roles of BEIIa and BEIIb in defining the structure of amylose and amylopectin were also examined in barley (Hordeum vulgare) endosperm. Barley lines with low expression of either BEIIa, BE IIb or both isoforms were generated through RNA-mediated silencing technology. These lines enabled the study of the role of each of these proteins in determining the amylose content, the distribution of chain lengths, and the frequency of branching in both amylose and amylopectin. A high amylose phenotype (> 70\%) was observed in lines 
expressing lower levels of BEIIa and BEIIb, while a reduction in the expression of either of these isoforms alone had minor impact on amylose content. The structure and properties of the barley high amylose starch resulting from the decrease in the expression of both BEII isoforms were found to be similar to those observed in amylose mutants of maize, which result from mutations that decrease the expression of the BEIIb gene. The analysis of amylopectin chain length distribution indicated that both BEIIa and BEIIb isoforms have distinct roles in determining the fine structure of amylopectin. A significant reduction in the frequency of branches in amylopectin was observed only when both BEIIa and BEIIb were reduced, whereas there was a significant increase in the branching frequency of amylose when BEIIb alone was reduced [61,65].

Other way of modifying amylase content is by SS expression. Amylose and amylopectin of rice mutants deficient in endosperm SS isoforms, either SSI ( $\Delta$ SSI) or SSIIIa ( $\triangle$ SSIIIa), were found to have an altered structure respect to to their parent (cv. Nipponbare, Np). The amylose content was higher in the mutants (Np, 15.5\%; $\Delta$ SSI, $18.2 \%$; $\Delta S I I I a, 23.6 \%$ ), and the molar ratio of branched amylose and its side chains was increased. In addition, the chainlength distribution of the $\beta$-amylase limit dextrins of amylopectin showed high regularity, which is consistent with the reported cluster structure. The mole $\%$ of the $\mathrm{B}(1)-\mathrm{B}(3)$ fractions was changed slightly in $\Delta$ SSI, which is consistent with the proposed role of SSI in elongating the external part of clusters. In $\triangle S S I I I a$, it has been observed a significant increase in the $\mathrm{B}(1)$ fraction and a decrease in both, the $\mathrm{B}(2)$ and $\mathrm{B}(3)$ fractions. The internal chain length of the $\mathrm{B}(2)$ and $\mathrm{B}(3)$ fractions appeared to be slightly altered, suggesting that the deficiency in SS affected the actions of branching enzyme(s) [66].

In another approach, SSIIIa null mutants of rice (Oryza sativa) were generated using retrotransposon insertion and chemical mutagenesis. The amylopectin $\mathrm{B}(2)$ to $\mathrm{B}(4)$ chains with degree of polymerization (DP) $>/=30$ and the $\mathrm{M}(\mathrm{r})$ of amylopectin were reduced to about $60 \%$ and $70 \%$ in the mutants, suggesting that SSIIIa plays an important role in the elongation of amylopectin $\mathrm{B}(2)$ to $\mathrm{B}(4)$ chains. Chains with DP 6 to 9 and DP 16 to 19 decreased while chains with DP 10 to 15 and DP 20 to 25 increased in the amylopectin mutants. These changes in the SSIIIa mutants are almost opposite images of those of SSIdeficient rice mutant and were caused by 1.3- to 1.7-fold increase of the amount of SSI in the mutant endosperm. Furthermore, the amylose content and the extralong chains (DP $>/=500$ ) of amylopectin were increased by 1.3- and 12-fold, respectively. These changes of starch composition of the mentioned mutants are due to the increase in about 1.7-fold of GBSSI activity. The starch granules of the mutants were found to be smaller with round shape and less crystalline. Thus, SSIIIa deficiency, the second major SS isoforrm in developing rice endosperm, affected either the structure of amylopectin, amylase content, and also the physicochemical properties of starch granules in two ways: directly by the SSIIIa deficiency itself and indirectly by up-regulation of both SSI and GBSSI mRNA [67].

By a different approach Safford et al [68] reported no effect on the amylose content of potato starch after the downregulation of the expression of the major branching enzyme isozyme (BE). However, a notable increase $(50-100 \%)$ of the phosphorous content was detected. 
Although the almost complete suppression of the branching enzyme activity (less than 5\% respect to wt levels) in transgenic potato tubers, no changes in amylose content of the starches derived from these transgenic lines were detected. Differences in the gelatinization properties (an increase of up to $5^{\circ} \mathrm{C}$ in the peak temperature and viscosity onset temperature) are reported, suggesting that these changes correlated with the branching pattern of the starch that result in changes of the double helix length. It is also possible that the increased phosphate content observed in the transgenic starches resulted in the elevation of the gelatinization temperature [68].

Other strategy to obtain high amylose starches was carried out by Itoh et al [60]. The Waxy (Wx) gene encodes a granule-bound starch synthase (GBSS) that plays a key role in the amylose synthesis of rice and other plant species. In rice, it has been described two functional Wx alleles: Wx(a), which produces higher amounts of amylose, and Wx(b), which produces low amounts of this polymer due to a mutation in the $5^{\prime}$ splicing site of intron 1 . When the $\mathrm{Wx}(\mathrm{a})$ cDNA was introduced into null-mutant Japonica rice $(\mathrm{wx})$ the amylose content were $6-11 \%$ higher than that of the original cultivar, Labelle, which carries the Wx(a) allele, although the levels of the Wx protein in the transgenic rice were equal to those of $\mathrm{cv}$. Labelle [60].

Finally, using A. thaliana null mutant lines for the SSIII locus, it has been postulated that SSIII has a regulatory role in the starch synthesis process [33]. These mutant lines show a higher accumulation of leaf starch during the day due to an apparent increase in biosynthetic rate. Besides, starch granules show physical alterations and higher phosphate content [33]. These data suggest that SSIII might have a negative regulatory role in starch synthesis. Previously, SSIII had been associated to a starch-excess phenotype, although indirectly through its association with regulatory proteins such as 14-3-3 [69]. In addition other SS isoform, SSIV, has been described to be essential for the initiation process of starch granule synthesis since A. thaliana SSIV mutant plants show just one large starch granule per plastid. The role of this isoform in the formation of the starch granule could be replaced in part by the SSIII isoform since the concomitant elimination of both enzymes in Arabidopsis block the starch synthesis. These data suggests that the remaining synthase activities are unable to start the synthesis of the starch granule. Recently, SSIV has been postulated to be also involved in the regulation of starch accumulation since its overexpression increases the starch levels in Arabidopsis leaves by $30 \%-40 \%$. In addition, SSIV-overexpressing lines display a higher growth rate. The increase in starch content as a consequence of enhanced SSIV expression is also observed in long-term storage starch organs such as potato tubers [70].

\section{Use of carbohydrate-binding modules to change amylose - amylopectin ratio and obtaining of modified starches.}

In the past few years the search for different strategies in order to produce starches with new properties was intensified. One of these strategies is to evaluate the possibility whether the microbial starch binding domains (SBDs) could be used as a universal tool for starch modification in plant biotechnology. 
It has been reported that SBDs are also present in microbial starch degrading enzymes. As mentioned above, one of the functions of SBD is to attach amylolytic enzymes to the insoluble starch granule. The amino acid sequences of these modules are very well conserved among different enzymes (i.e. glucoamylase, $\alpha$-amylase, $\beta$-amylase, etc.), as well as among different species such as Clostridium thermosulfurogenes, Bacillus circulans, Aspergillus niger, Klebsiella pneumonia, Streptomyces limosus, Pseudomonas stutzeri, etc. [50,7173]. Several studies have shown that these enzymes lose (most of) their catalytic activity towards raw starch granules upon removal of the SBD, whereas their activity on soluble substrates remains unaltered. Besides their affinity for starch granules, SBDs can also bind maltodextrins and cyclodextrins [71]. Ji et al [72], explored the possibility of engineering artificial granule-bound proteins, which can be incorporated in the granule during biosynthesis. The SBD-encoding region of cyclodextrin glycosyltransferase from $B$. circulans was fused to the sequence encoding the transit peptide (amyloplast entry) of potato GBSSI. The synthetic gene was expressed in the tubers of two potato cultivars and one amylose-free (amf) potato mutant. The results showed that SBDs are accumulated inside starch granules, not at the granule surface and amylose-free granules contained 8 times more SBD than the amylose-containing ones. However, no consistent differences in physicochemical properties between transgenic SBD starches and their corresponding controls were found, suggesting that SBD can be used as an anchor for effector proteins without having side-effects [72].

On the other hand it was also evaluated whether is it possible to produce an amylose-free potato starch by displacing GBSSI, from the starch granule by engineering multiple-repeat CBM20 SBD (two, three, four and five). The constructs were introduced in wild type potato cultivar, and the starches of the resulting transformants were compared with those expressing amf potato clones. The amount of SBDs accumulated in starch granules was increased progressively from SBD to SBD3 and not when were used SBD4 and SBD5; however, a reduction in amylose content was not achieved in any of the transformants. It was shown that SBDn expression can affect the physical process underlying granule assembly in both potato genetic backgrounds, without altering the primary structure of the constituent starch polymers and the granule melting temperature. Granule size distribution of the starches obtained from transgenic plants was similar to untransformed controls, irrespective of the amount of SBDn accumulated. In the amf background, granule size is severely affected [74].

In the case of starches which require chemical modifications to enhance their properties, such as the improved stability in solution by acetylation, a drawback is generated when pollutant chemicals are used. A biological alternative to the derivatization process was investigated by the expression of an amyloplast-targeted Escherichia coli maltose acetyltransferase (MAT) in tubers of wild-type and mutant amf potato plants. MAT was expressed alone, or fused in its $\mathrm{N}$ - or C-terminus to a SBD to be target to the starch granule. Starch granules derived from transgenic plants contained acetyl groups in low number. In addition, MAT protein on the starch granules present catalytic activity even after postharvesting, when supplied with glucose or maltose and acetyl-coenzyme A, but it was not able to acetylate starch polymers in vitro. Starch granules from transformants where MAT 
was expressed alone also showed MAT catalytic activity, indicating that MAT is accumulated in starch granules, and could bind to the polymer without the presence of any SBD. Furthermore, the fusion of MAT and SBD affects granule morphology: in potato transformants, the percentage of altered granules when the SBD was located at the Cterminal end correlated with the amount of fusion protein accumulated. When SBD was located at the N-terminus of MAT or it is absent, no differences were found respect to the untransformed controls, indicating that not only is the simultaneous presence of SBD and MAT important for altering granule morphology, but also their localization in the fusion protein [75].

Another approach to obtain modified starches involves the bacterial glucansucrases [76]. Certain bacteria possess an array of enzymes, so-called glucansucrases, which can attach (contiguous) 1,6-linked or 1,3-linked glucosyl residues to maltodextrins. This, together with the presence of sucrose inside the potato tuber amyloplast [77], suggests that glucansucrases are of great interest for diversifying starch structure. With few exceptions, glucansucrases are extracellular enzymes, which are produced by lactic acid bacteria such as Leuconostoc mesenteroides, oral Streptococci and some species of Lactococcus and Lactobacillus [78]. The glucansucrases catalyze the polymerization of glucose residues from sucrose, which leads to the production of a large variety of $\alpha$-glucans with different sizes and structures, and composed of diverse linkage types. Most glucansucrases share a common structure composed of four different regions: a signal peptide, a variable region, a catalytic domain, and a glucan-binding domain (GBD) [76].

Production of water-insoluble mutan polymers in wild type potato tubers was investigated by Kok-Jacon et al (2005) after expression of full-length GTFI (mutansucrase) and a truncated version without glucan-binding domain from Streptococcus downei. Mutan polymers are bacterial polysaccharides that are secreted by oral microorganisms and have adhesive properties and different degrees of water-solubility [81]. They account for about $70 \%$ of the carbohydrates present in dental plaque [79] in addition to dextrans and levans [80]. When the short form of the protein was expressed, low amounts of mutan polymer attached to the starch granules has been detected. Besides, these plants exhibited severely altered tuber phenotype and starch granule morphology in comparison to those expressing the full-length GTFI gene, whereas no changes at the starch level were observed. Finally, the rheological properties of the starch obtained from plants expressing the truncated protein were also altered, showing a higher retrogradation during cooling of the starch paste [80].

Subsequently, the same group of investigators fused the truncated form of a mutansucrase (without glucan binding domain) to an N- or C- terminal SBD. The different enzymes were introduced into two genetically different potato backgrounds (wild type and amf lines), in order to attach the enzyme to the growing starch granules, and to facilitate the incorporation of mutan polymers in starch. Starches from the chimeric transformants seemed to contain less amounts of mutan than those from plants expressing the mutansucrase alone, suggesting that SBD might inhibit the catalytic activity of the enzyme. Scanning electron microscopy showed that expression of SBD-mutansucrase fusion proteins resulted in alterations of granule morphology in both genetic backgrounds. Surprisingly, the amf 
starches containing the chimeric form had a spongeous appearance, as the granule surface contained many small holes and grooves, indicating that this fusion protein can interfere with the lateral interactions of amylopectin sidechains. No differences in physicochemical properties of the transgenic starches were observed [82].

Finally, all the knowledge gained about the characteristics, structure, function and occurrence of SBD and GBD will support current and future experimental research. Since SBD are domains which retain their structural fold and functional properties independently of the remaining parts of the protein molecule including the catalytic domain, they can be applied in various fields of biotechnology [48,83-86]. It is important to note that most of the applications have involved only the CBM20 SBD. One of the most attractive fields is represented by starch processing in the food industry, especially the hydrolysis of starch into maltodextrins and maltooligosaccharides [87]. Since conventional processes require starch gelatinization at elevated temperature and thus use of thermostable amylolytic enzymes [88], the possibility of carrying out the processes without gelatinization, by utilizing new enzymes with attached SBD is desirable $[52,89,90]$.

\section{Conclusions}

Food production in terms of quality and quantity, as well as for new plants commodities and products in developed and developing countries, cannot based only on classical agriculture [91]. The metabolic engineering of plants has yielded remarkable results by increasing the production of minor components (essential oils, vitamin A, vitamin E and flavonoids) and, as well as the composition of major components, such as starch or fatty acids [92]. The improvement in the food we eat is necessary and crucial in societies that have bad eating habits. The health benefits provided by the intake of resistant starches have been properly tested and it will be desirable that these kinds of starches could be incorporated into the human diet. Molecular tools available at the present and those likely to be developed in the near future, will enable the development of new strategies to increase the content of resistant starch in grains and other vegetables. Manipulation of the starch synthesis pathway through the modification of enzymes belonging to this route, and the use of CBM (and specifically $\mathrm{SBD}$ ) of both microbial and plant, are alternatives that are desirable to explore in more detail.

\section{Author details}

María Victoria Busi and Diego F. Gomez-Casati

Centro de Estudios Fotosintéticos y Bioquímicos (CEFOBI-CONICET),

Suipacha 570, Rosario, Argentina

IIB-INTECH, Universidad Nacional de General San Martín (UNSAM), San Martín, Buenos Aires, Argentina

Mariana Martín

Centro de Estudios Fotosintéticos y Bioquímicos (CEFOBI-CONICET), Universidad Nacional de Rosario, Suipacha 570, Rosario, Argentina 


\section{Acknowledgement}

Our work is supported in part by grants from the Biotechnology Program from Universidad Nacional de General San Martin (UNSAM) (PROG07F / 2-2007), Consejo Nacional de Investigaciones Científicas y Técnicas (CONICET, PIP 00237) and Agencia Nacional de Promoción Científica y Tecnológica (ANPCyT, PICT 2010 - 0543 and PICT 2010 - 0069). MVB, MM and DGC are research members from CONICET.

\section{References}

[1] Halpin, C. (2005). Gene stacking in transgenic plants--the challenge for 21st century plant biotechnology. Plant Biotechnol J 3, 141-55.

[2] Cassidy, A. (2004) Serials, Nuts and Pulses. In Plants: Diet and health (Goldberg, G., ed.), pp. 134-146. Blackwell Sciences, Oxford, UK.

[3] Nugent, A.P. (2005). Health properties of resistant starch. Nutrition Bull 30

[4] Zhao, F.J. and Shewry, P.R. (2010). Recent developments in modifying crops and agronomic practice to improve human health Food Policy

[5] Lopez, X. (2009). Obesity: How did resistant starches might contribute? Enfasis Alim 2, 32-38.

[6] Dominguez Vergara, A.M., Vazquez-Moreno, L. and Ramos-Clamont Mon Fort, G. (2009). Review of the role of prebiotic oligosaccharides in the prevention of gastrointestinal infections. Arch Latin Nutr 59, 358-368.

[7] Topping, D.L., Fukushima, M. and Bird, A.R. (2003). Resistant starch as a prebiotic and synbiotic: state of the art. Proc Nutr Soc 62, 171-6.

[8] Robertson, M.D. (2012). Dietary-resistant starch and glucose metabolism. Curr Opin Clin Nutr Metab Care

[9] Shamaia, K., Bianco-Peled, H. and Shimonic, E. (2003). Polymorphism of resistant starch type III Carbohydr Polym 54, 363-369.

[10] Morell, M.K., Konik-Rosse, C., Ahmed, R., Li, Z. and Rahman, S. (2004). Synthesis of resistant starch in plants. J AOAC Int 87, 740-748.

[11] Bird, A.R., Flory, C., Davies, D.A., Usher, S. and Topping, D.L. (2004). A novel barley cultivar (Himalaya 292) with a specific gene mutation in starch synthase IIa raises large bowel starch and short-chain fatty acids in rats. J Nutr 134, 831-5.

[12] Higgins, J.A., Higbee, D.R., Donahoo, W.T., Brown, I.L., Bell, M.L. and Bessesen, D.H. (2004). Resistant starch consumption promotes lipid oxidation. Nutr Metab (Lond) 1, 8.

[13] Farhat, I.A., Protzmann, J., Becker, A., Valles-Pamies, B., Neale, R. and Hill, S.E. (2001). Effect of the extent of conversion and retrogradation on the digestibility of potato starch. Starch/Stärke 53, 431-436.

[14] Sivak, M. and Preiss, J. (1998). Advances in Food Nutrition Research. Academic Press, San Diego, California. 41

[15] Alonso, A.G., Calixto, F.S. and Delcour, J.A. (1998). Influence of botanical source and proccesing on formation of resistant starch type III. Cereal Chem 75, 802-804. 
[16] Thompson, D.B. (2000). Strategies for the manufacture of resistant starch. Trends Food Sci Technol 11, 245-253.

[17] Jenner, H.L. (2003). Transgenesis and yield: what are our targets? Trends Biotechnol 21, 190-2.

[18] Willmitzer, L. (1999). Plant Biotechnology: Output traits-the second generation of plant biotechnology products is gaining momentum. Curr Opin Biotechnol 10, 161-162.

[19] Morell, M.K. et al. (2003). Barley sex6 mutants lack starch synthase IIa activity and contain a starch with novel properties. Plant J 34, 173-85.

[20] Blauth, S.L., Kim, K.N., Klucinec, J., Shannon, J.C., Thompson, D. and Guiltinan, M. (2002). Identification of Mutator insertional mutants of starch-branching enzyme 1 (sbe1) in Zea mays L. Plant Mol Biol 48, 287-97.

[21] Gao, M., Fisher, D.K., Kim, K.N., Shannon, J.C. and Guiltinan, M.J. (1997). Independent genetic control of maize starch-branching enzymes IIa and IIb. Isolation and characterization of a Sbe2a cDNA. Plant Physiol 114, 69-78.

[22] Roach, P.J. (2002). Glycogen and its metabolism. Curr Mol Med 2, 101-20.

[23] Dauvillee, D. et al. (2009). Genetic dissection of floridean starch synthesis in the cytosol of the model dinoflagellate Crypthecodinium cohnii. Proc Natl Acad Sci U S A 106, 21126-30.

[24] Cavalier-Smith, T. (2009). Predation and eukaryote cell origins: a coevolutionary perspective. Int J Biochem Cell Biol 41, 307-22.

[25] Patron, M.J. and Keeling, P. (2005). Common evolutionary origin of starch biosynthetic enzymes in green and red algae. J Phycol 41, 1131-1141.

[26] Deschamps, P., Moreau, H., Worden, A.Z., Dauvillee, D. and Ball, S.G. (2008). Early gene duplication within chloroplastida and its correspondence with relocation of starch metabolism to chloroplasts. Genetics 178, 2373-87.

[27] Deschamps, P., Haferkamp, I., d'Hulst, C., Neuhaus, H.E. and Ball, S.G. (2008). The relocation of starch metabolism to chloroplasts: when, why and how. Trends Plant Sci $13,574-82$.

[28] Tetlow, I.J. (2011). Starch biosynthesis in developing seeds. Seed Sci Res 21, 5-32.

[29] Smith, A.M., Denyer, K. and Martin, C. (1997). The Synthesis of the Starch Granule. Annu Rev Plant Physiol Plant Mol Biol 48, 67-87.

[30] Ball, S.G. and Morell, M.K. (2003). From bacterial glycogen to starch: understanding the biogenesis of the plant starch granule. Annu Rev Plant Biol 54, 207-33.

[31] Delvalle, D. et al. (2005). Soluble starch synthase I: a major determinant for the synthesis of amylopectin in Arabidopsis thaliana leaves. Plant J 43, 398-412.

[32] Maddelein, M.L. et al. (1994). Toward an understanding of the biogenesis of the starch granule. Determination of granule-bound and soluble starch synthase functions in amylopectin synthesis. J Biol Chem 269, 25150-7.

[33] Zhang, X., Myers, A.M. and James, M.G. (2005). Mutations affecting starch synthase III in Arabidopsis alter leaf starch structure and increase the rate of starch synthesis. Plant Physiol 138, 663-74. 
[34] Delrue, B. et al. (1992). Waxy Chlamydomonas reinhardtii: monocellular algal mutants defective in amylose biosynthesis and granule-bound starch synthase activity accumulate a structurally modified amylopectin. J Bacteriol 174, 3612-20.

[35] Wattebled, F., Planchot, V., Dong, Y., Szydlowski, N., Pontoire, B., Devin, A., Ball, S. and D'Hulst, C. (2008). Further evidence for the mandatory nature of polysaccharide debranching for the aggregation of semicrystalline starch and for overlapping functions of debranching enzymes in Arabidopsis leaves. Plant Physiol 148, 1309-23.

[36] Coppin, A. et al. (2005). Evolution of plant-like crystalline storage polysaccharide in the protozoan parasite Toxoplasma gondii argues for a red alga ancestry. J Mol Evol 60, 257-67.

[37] Ritte, G., Heydenreich, M., Mahlow, S., Haebel, S., Kotting, O. and Steup, M. (2006). Phosphorylation of C6- and C3-positions of glucosyl residues in starch is catalysed by distinct dikinases. FEBS Lett 580, 4872-6.

[38] Baunsgaard, L., Lutken, H., Mikkelsen, R., Glaring, M.A., Pham, T.T. and Blennow, A. (2005). A novel isoform of glucan, water dikinase phosphorylates pre-phosphorylated alpha-glucans and is involved in starch degradation in Arabidopsis. Plant J 41, 595-605.

[39] Kotting, O., Pusch, K., Tiessen, A., Geigenberger, P., Steup, M. and Ritte, G. (2005). Identification of a novel enzyme required for starch metabolism in Arabidopsis leaves. The phosphoglucan, water dikinase. Plant Physiol 137, 242-52.

[40] Kotting, O., Kossmann, J., Zeeman, S.C. and Lloyd, J.R. (2010). Regulation of starch metabolism: the age of enlightenment? Curr Opin Plant Biol 13, 321-9.

[41] Myers, A.M., Morell, M.K., James, M.G. and Ball, S.G. (2000). Recent progress toward understanding biosynthesis of the amylopectin crystal. Plant Physiol 122, 989-97.

[42] Blennow, A. and Svensson, B. (2010). Dynamics of starch granule biogenesis -the role of redox regulated enzymes- and low-affinity carbohydrate binding modules. Biocatal Biotransf 28, 3-9.

[43] Rahman, S. et al. (2003). The sugary-type isoamylase gene from rice and Aegilops tauschii: characterization and comparison with maize and arabidopsis. Genome 46, 496506.

[44] Morell, M.K. and Myers, A.M. (2005). Towards the rational design of cereal starches. Curr Opin Plant Biol 8, 204-10.

[45] Gilkes, N.R., Warren, R.A., Miller, R.C., Jr. and Kilburn, D.G. (1988). Precise excision of the cellulose binding domains from two Cellulomonas fimi cellulases by a homologous protease and the effect on catalysis. J Biol Chem 263, 10401-7.

[46] Tomme, P., Driver, D.P., Amandoron, E.A., Miller, R.C., Jr., Antony, R., Warren, J. and Kilburn, D.G. (1995). Comparison of a fungal (family I) and bacterial (family II) cellulose-binding domain. J Bacteriol 177, 4356-63.

[47] Tomme, P., Warren, R.A. and Gilkes, N.R. (1995). Cellulose hydrolysis by bacteria and fungi. Adv Microb Physiol 37, 1-81.

[48] Shoseyov, O., Shani, Z. and Levy, I. (2006). Carbohydrate binding modules: biochemical properties and novel applications. Microbiol Mol Biol Rev 70, 283-95.

[49] Boraston, A.B., Bolam, D.N., Gilbert, H.J. and Davies, G.J. (2004). Carbohydrate-binding modules: fine-tuning polysaccharide recognition. Biochem J 382, 769-81. 
[50] Machovic, M. and Janecek, S. (2006). Starch-binding domains in the post-genome era. Cell Mol Life Sci

[51] Machovic, M. and Janecek, S. (2006). The evolution of putative starch-binding domains. FEBS Lett 580, 6349-56.

[52] Janecek, S., Svensson, B. and MacGregor, E.A. (2011). Structural and evolutionary aspects of two families of non-catalytic domains present in starch and glycogen binding proteins from microbes, plants and animals. Enzyme Microb Technol 49, 429-440.

[53] Wayllace, N.Z., Valdez, H.A., Ugalde, R.A., Busi, M.V. and Gomez-Casati, D.F. (2010). The starch-binding capacity of the noncatalytic SBD2 region and the interaction between the $\mathrm{N}$ - and $\mathrm{C}$-terminal domains are involved in the modulation of the activity of starch synthase III from Arabidopsis thaliana. Febs J 277, 428-40.

[54] Valdez, H.A., Peralta, D.A., Wayllace, N.Z., Grisolía, M.J., Gomez-Casati, D.F. and Busi, M.V. (2011). Preferential binding of SBD from Arabidopsis thaliana SSIII to polysaccharides. Study of amino acid residues involved. Starch/Stärke 63, 451-460.

[55] Southall, S.M., Simpson, P.J., Gilbert, H.J., Williamson, G. and Williamson, M.P. (1999). The starch-binding domain from glucoamylase disrupts the structure of starch. FEBS Lett 447, 58-60.

[56] Kerk, D., Conley, T.R., Rodriguez, F.A., Tran, H.T., Nimick, M., Muench, D.G. and Moorhead, G.B. (2006). A chloroplast-localized dual-specificity protein phosphatase in Arabidopsis contains a phylogenetically dispersed and ancient carbohydrate-binding domain, which binds the polysaccharide starch. Plant J 46, 400-13.

[57] Roma-Mateo, C. et al. (2011). Laforin, a dual specificity protein phosphatase involved in Lafora disease, is phosphorylated at Ser25 by AMP-activated protein kinase. Biochem J

[58] Palopoli, N., Busi, M.V., Fornasari, M.S., Gomez-Casati, D., Ugalde, R. and Parisi, G. (2006). Starch-synthase III family encodes a tandem of three starch-binding domains. Proteins 65, 27-31.

[59] Valdez, H.A., Busi, M.V., Wayllace, N.Z., Parisi, G., Ugalde, R.A. and Gomez-Casati, D.F. (2008). Role of the N-terminal starch-binding domains in the kinetic properties of starch synthase III from Arabidopsis thaliana. Biochemistry 47, 3026-32.

[60] Itoh, K., Ozaki, H., Okada, K., Hori, H., Takeda, Y. and Mitsui, T. (2003). Introduction of Wx transgene into rice wx mutants leads to both high- and low-amylose rice. Plant Cell Physiol 44, 473-80.

[61] Jobling, S.A., Westcott, R.J., Tayal, A., Jeffcoat, R. and Schwall, G.P. (2002). Production of a freeze-thaw-stable potato starch by antisense inhibition of three starch synthase genes. Nat Biotechnol 20, 295-9.

[62] Regina, A. et al. (2006). High-amylose wheat generated by RNA interference improves indices of large-bowel health in rats. Proc Natl Acad Sci U S A 103, 3546-51.

[63] Sestili, F., Janni, M., Doherty, A., Botticella, E., D'Ovidio, R., Masci, S., Jones, H.D. and Lafiandra, D. (2010). Increasing the amylose content of durum wheat through silencing of the SBEIIa genes. BMC Plant Biol 10, 144.

[64] Butardo, V.M. et al. (2011). Impact of down-regulation of starch branching enzyme IIb in rice by artificial microRNA- and hairpin RNA-mediated RNA silencing. J Exp Bot 62, 4927-41. 
[65] Regina, A., Kosar-Hashemi, B., Ling, S., Li, Z., Rahman, S. and Morell, M. (2010). Control of starch branching in barley defined through differential RNAi suppression of starch branching enzyme IIa and IIb. J Exp Bot 61, 1469-82.

[66] Hanashiro, I., Higuchi, T., Aihara, S., Nakamura, Y. and Fujita, N. (2011). Structures of starches from rice mutants deficient in the starch synthase isozyme SSI or SSIIIa. Biomacromolecules 12, 1621-8.

[67] Fujita, N. et al. (2007). Characterization of SSIIIa-deficient mutants of rice: the function of SSIIIa and pleiotropic effects by SSIIIa deficiency in the rice endosperm. Plant Physiol 144, 2009-23.

[68] Safford, R.E. et al. (1998). Consequences of antisense RNAi of starch branching enzyme activity on properties of potato starch. Carbohydr Polym 35, 155-160.

[69] Sehnke, P.C., Chung, H.J., Wu, K. and Ferl, R.J. (2001). Regulation of starch accumulation by granule-associated plant 14-3-3 proteins. Proc Natl Acad Sci U S A 98, 765-70.

[70] Szydlowski, N. et al. (2009). Starch granule initiation in Arabidopsis requires the presence of either class IV or class III starch synthases. Plant Cell 21, 2443-57.

[71] Svensson, B., Jespersen, H., Sierks, M.R. and MacGregor, E.A. (1989). Sequence homology between putative raw-starch binding domains from different starchdegrading enzymes. Biochem J 264, 309-11.

[72] Ji, Q., Vincken, J.P., Suurs, L.C. and Visser, R.G. (2003). Microbial starch-binding domains as a tool for targeting proteins to granules during starch biosynthesis. Plant Mol Biol 51, 789-801.

[73] Janecek, S. and Sevcik, J. (1999). The evolution of starch-binding domain. FEBS Lett 456, 119-25.

[74] Firouzabadi, F.N., Vincken, J.P., Ji, Q., Suurs, L.C., Buleon, A. and Visser, R.G. (2007). Accumulation of multiple-repeat starch-binding domains (SBD2-SBD5) does not reduce amylose content of potato starch granules. Planta 225, 919-33.

[75] Nazarian Firouzabadi, F., Vincken, J.P., Ji, Q., Suurs, L.C. and Visser, R.G. (2007). Expression of an engineered granule-bound Escherichia coli maltose acetyltransferase in wild-type and amf potato plants. Plant Biotechnol J 5, 134-45.

[76] Kok-Jacon, G.A., Ji, Q., Vincken, J.P. and Visser, R.G. (2003). Towards a more versatile alpha-glucan biosynthesis in plants. J Plant Physiol 160, 765-77.

[77] Gerrits, N., Turk, S.C., van Dun, K.P., Hulleman, S.H., Visser, R.G., Weisbeek, P.J. and Smeekens, S.C. (2001). Sucrose metabolism in plastids. Plant Physiol 125, 926-34.

[78] Robyt, J.F. (1995). Mechanisms in the glucansucrase synthesis of polysaccharides and oligosaccharides from sucrose. Adv Carbohydr Chem Biochem 51, 133-68.

[79] Loesche, W.J. (1986). Role of Streptococcus mutans in human dental decay. Microbiol Rev 50, 353-80.

[80] Kok-Jacon, G.A., Vincken, J.P., Suurs, L.C. and Visser, R.G. (2005). Mutan produced in potato amyloplasts adheres to starch granules. Plant Biotechnol J 3, 341-51.

[81] Sutherland, I. (2001). Biofilm exopolysaccharides: a strong and sticky framework. Microbiology 147, 3-9. 
[82] Nazarian Firouzabadi, F., Kok-Jacon, G.A., Vincken, J.P., Ji, Q., Suurs, L.C. and Visser, R.G. (2007). Fusion proteins comprising the catalytic domain of mutansucrase and a starch-binding domain can alter the morphology of amylose-free potato starch granules during biosynthesis. Transgenic Res 16, 645-56.

[83] Juge, N. et al. (2006). The activity of barley alpha-amylase on starch granules is enhanced by fusion of a starch binding domain from Aspergillus niger glucoamylase. Biochim Biophys Acta 1764, 275-84.

[84] Ohdan, K., Kuriki, T., Takata, H., Kaneko, H. and Okada, S. (2000). Introduction of raw starch-binding domains into Bacillus subtilis alpha-amylase by fusion with the starchbinding domain of Bacillus cyclomaltodextrin glucanotransferase. Appl Environ Microbiol 66, 3058-64.

[85] Latorre-Garcia, L., Adam, A.C., Manzanares, P. and Polaina, J. (2005). Improving the amylolytic activity of Saccharomyces cerevisiae glucoamylase by the addition of a starch binding domain. J Biotechnol 118, 167-76.

[86] Christiansen, C., Abou Hachem, M., Janecek, S., Vikso-Nielsen, A., Blennow, A. and Svensson, B. (2009). The carbohydrate-binding module family 20--diversity, structure, and function. Febs J 276, 5006-29.

[87] Van Geel-Schutten, G.H. et al. (1999). Biochemical and structural characterization of the glucan and fructan exopolysaccharides synthesized by the lactobacillus reuteri wildtype strain and by mutant strains. Appl Environ Microbiol 65, 3008-14.

[88] Bertoldo, C. and Antranikian, G. (2002). Starch-hydrolyzing enzymes from thermophilic archaea and bacteria. Curr Opin Chem Biol 6, 151-60.

[89] Vikso-Nielsen, A., Andersen, C., Hoff, T. and Pedersen, S. (2006). Development of new alpha-amylases for raw starch hydrolysis. Biocatal Biotransf 24, 121-127.

[90] Wang, P., Singh, V., Xue, H., Johnston, D.B., Rausch, K.D. and Tumbleson, M.E. (2007). Comparisson of raw starch hydrolysis enzyme with conventional liquetfaction and saccharification enzymes in dry-grind corn proccesing. Cereal Chem 84, 10-14.

[91] Altman, A. (1999). Plant Biotechnology in the 21st century: the challenges ahead. Electr J Biotechnol 2, 51-55.

[92] Morandini, P. (2009). Rethinking metabolic control. Plant Sci 176, 441-451. 